neurodisability disease from April 2017-September 2018 were identified for audit. Their medical records were reviewed to determine if these locally used prognostic indicators had been used and if appropriate interventions from the EoL care prompt had been recorded as completed.

Results 7 locally used prognostic indicators were determined from interviews. Each of the 20 patients identified for audit had evidence of at least 1 of these in their notes. Interventions from the EoL care prompt were completed best in the last few days of life, however only 1 patient met the standard of completion of all interventions across the 3 sections.

Conclusions Prognostic indicators are being used in the last few days of life, and these could be added to the EoL care prompt to improve its use. Despite this, not all interventions for EoL care are being completed. Further research is required to determine useful prognostic indicators and additionally to develop an effective Children's EoL care planning tool, which would be an exciting future for improving children's EoL care.

\section{G365(P) IMPROVING PREPARATION AND SUPPORT FOR PARENTS WHO CARE FOR CHILDREN WITH MEDICAL COMPLEXITY AT HOME}

${ }^{1} \mathrm{~B}$ Page, ${ }^{1} \mathrm{C}$ Vincent, ${ }^{2} \mathrm{~A}$ Lee, ${ }^{3} \mathrm{E}$ Harrop, ${ }^{4} \mathrm{~L}$ Hinton, ${ }^{1} \mathrm{~N}$ Yeung. ${ }^{1}$ Department of Experimental Psychology, University of Oxford, Oxford, UK; ${ }^{2}$ Oxford University Hospitals NHS Trust, Oxford, UK; ${ }^{3}$ Helen and Douglas House, Oxford, UK; ${ }^{4}$ Department of Primary Care, University of Oxford, Oxford, UK

\subsection{6/archdischild-2020-rcpch.313}

Aims There are increasing numbers of children who require complex medical care at home, (e.g. tube feeding and tracheostomy care). Parents, with the support of nurses and other carers, deliver the day-to-day care these children require. There are many benefits of care at home for the child and family but also some serious risks. The aim of this research is to investigate the problems occurring with complex care for children at home, and to develop interventions to help better prepare and support parents to provide safe medical care to their children.

Methods We are using a mixture of clinical and experimental studies to explore the safety problems, and to test and develop interventions. Methods include analysis of incident reports, thematic analysis of interviews and surveys with parents, and mixed-methods analysis of interventions.

Results The findings highlight a range of safety problems occurring with complex care at home, many of which can remain hidden from healthcare services. In interviews with parents, the key themes identified were responsibilities of the parents, impact on the family and change over time. Many parents reported feeling overwhelmed by their responsibilities. In our analysis of incident reports on feeding tube care and long-term ventilation at home, the most commonly reported concerns related to broken equipment and inadequate training for parents, nurses and carers. Experiments on feeding tube training suggest that training that is easy to understand may make parents over-confident and reduce the time they spend studying, leading to worst performance in a test of their knowledge. In a survey, parents indicated that simulation training and videos may be helpful methods of learning and we are exploring this further.
Implications This research shows that the provision of services to support parents caring for children with complex needs is highly variable. Good quality training for parents and carers is vital if these children are to be cared for safely at home. There needs to be more investment and system-wide changes to training and support for parents and carers who provide complex care at home.

\section{G366(P) CHALLENGES AND FACILITATORS TO JOINT-WORKING AND REFERRAL TO PAEDIATRIC PALLIATIVE CARE: A HEALTHCARE PROFESSIONAL PERSPECTIVE}

${ }^{1,2,3} \mathrm{C}$ Holland, ${ }^{1,2}$ E Harrop. ' General Paediatrics, John Radcliffe University Hospital, Oxford, UK; ${ }^{2}$ Paediatric Palliative Care, Helen and Douglas House Children's Hospice, Oxford, UK; ${ }^{3}$ University School of Medicine, Cardiff University, Cardiff, UK

\subsection{6/archdischild-2020-rcpch.314}

Background Despite the National Institute of Clinical Excellence suggesting involvement of specialist paediatric palliative care (PPC) leads to better symptom control and 'total care' of patients/families for those with life-limited or lifethreatening conditions, many of those eligible are not referred.

Aim To explore healthcare professionals views on challenges and facilitators for referral to PPC, in a tertiary hospital with no integrated palliative care team.

This research will help inform future quality improvement projects locally, to ensure more children's palliative care needs are met.

Methods Initial literature review to direct qualitative research: Medline search using terms associated with 'healthcare professionals', 'paediatric palliative care' and 'referral', screened for relevance.

Qualitative research Focus groups (approximately 5-8 healthcare professionals sufficiently senior to refer to PPC and voluntarily consenting to take part) will be held for each department area. Departments are grouped due to clinical cross-over and to ensure appropriately sized focus groups but aim to span the full spectrum of PPC needs, including neonatal, intensive care, general and community paediatrics and neurosurgery amongst other sub-specialties.

Participants' views, knowledge and current practices around referral to PPC will be explored, through fictitious case vignettes of children with different PPC needs.

Thematic analysis of recorded audio of earlier focus groups will inform approach/question style for subsequent. Differences between departments will also be compared.

Results Current literature is often US-based or uses oncology/ intensive care cases, therefore not generalisable to UK-based children across the spectrum of PPC needs. Twamley et al (2014) had most relevance, however, leaves a gap in examining differences between sub-specialties and suggests 'deeply embedded attitudes' may be the reason for discordance between understanding and referral rates.

The qualitative research is currently being undertaken. We expect to present preliminary results from focus groups held from February 2020.

Conclusions The researchers hope to draw themes from the focus groups. We expect some overarching themes throughout all groups but others that may be more department specific. We are interested to see how health professionals perceive palliative care needs for case vignettes that are somewhat removed from their usual caseload. 